S. Koizumi

Nagoya Math. J.

Vol. 48 (1972), 37-55

\title{
THE FIELDS OF MODULI FOR POLARIZED ABELIAN VARIETIES AND FOR CURVES
}

\author{
SHOJI KOIZUMI
}

In the study of moduli of polarized abelian varieties and of curves as well as in the theory of complex multiplications, the notion of fields of moduli for structures plays an essential role. This notion was first introduced by Matsusaka [7] for polarized varieties with some pleasing properties and later was given a more comprehensible treatment by Shimura [10] in the case of polarized abelian varieties or polarized abelian varieties with some further structures. Both authors discussed fields of moduli not only in algebraic geometry of characteristic zero but also in that of positive characteristic, but in the latter case the definition of fields of moduli seems somewhat artificial and there have been no essential applications of them so far.

The purpose of the present paper is, despite our pretensions to achieve generality in $\S 1$, to prove the following fact, which is known in geometry of characteristic zero.

( I) Assume that the characteristic of the universal domain is not two. Let $S$ be a polarized abelian variety or a complete non-singular curve; then the field of moduli for $S$ coincides with the intersection of all possible fields of rationality for all possible structures isomorphic to $S$. (Corollary 3.2.2)

We have followed the definition of "field of moduli" given by Matsusaka and Shimura in the above statement, but in this paper the fields of moduli are defined in a somewhat different way and hence the expression of our Corollary is adjusted to our definition. We shall now give a summary of the contents of this paper.

After giving some fundamental notations we recall some properties of a Picard variety of a complete variety with no divisorial singularities, which are used in Example 1.2.3 and in the proof of Proposition 3.1.

Received October 1, 1971. 
In this part we followed the notations and formulations in Lang's book [3] as a general principle, where we find proofs of all our statements except the duality theorem of abelian varieties (i.e., the fact that the map $\kappa_{A}$ is an isomorphism), which actually is not used in the paper $(\S 0$.). In $\S 1$, we start by defining $F M$-structures $S$ for which the notions of fields of rationality, the transform $S^{\sigma}$ by an automorphism $\sigma$ of the universal domain and the isomorphy relation are given with very natural axioms $(\mathrm{fm})$. We then define the notion of the "field of moduli for $S$ ", which is different from the old definition in appearance but will be shown the same in the case of polarized abelian varieties. By several examples we try to explain how our notion works in algebraic geometry. Before studying polarized varieties we consider an $\mathscr{A}$-structure $\mathfrak{A}$ consisting of a complete non-singular variety and an algebraic equivalence class on it satisfying some conditions (see 2.1) and see the existence of the field of moduli for $\mathfrak{A}$ under a condition (A) (see 2.1). This is discussed in $\S 2$ and since that section does not include any new results, most proofs are omitted or only sketched. In $\S 3$, we prove the existence of the field of moduli for a polarized variety under the assumptions $(T)$ and $(P)$ (see Theorem 3.2). These assumptions seem too strong for general theory of polarized varieties, though they are general enough for our purpose as far as we are concerned with polarized abelian varieties or curves. Using our new definition of "fields of moduli" the statement (I) can be rewritten in the following way: ( $\left.\mathrm{I}^{\prime}\right)$ The assumptions and $S$ being as in I), the field of moduli for $S$ in our sense exists and it coincides with the old field of moduli for $S$ (in spite of their difference in appearance). Statement (I) is reduced to a special case of Theorem 3.2 on account of Proposition 3.1.

\section{§ 0 . Terminology and notations.}

We follow the terminology and definitions in Weil $[13,14]$. Throughout the paper the universal domain is fixed and denoted by $\boldsymbol{K}$. Any field we consider is a subfield of $K$, and a variety is an absolutely irreducible variety rational over a field. For a field $F$, Aut $(K / F)$ means the subgroup of the automorphism group Aut $(K)$ of $K$ consisting of elements acting as the identity of $F$.

Let $V$ be a complete variety non-singular in codimension 1 ; then we denote by $\mathscr{D}(V), \mathscr{D}_{a}(V)$ and $\mathscr{D}_{\ell}(V)$, respectively the group of $V$-divisors, 
the group of $V$-divisors algebraically equivalent to zero and the group of $V$-divisors linearly equivalent to zero. For a divisor $X \in \mathscr{D}(V)$, the linear equivalence class determined by $X$ is denoted by $C l(X)$.

When we regard an abelian variety $A$ as a variety without consideration of its composition law, we call it the underlying variety of the abelian variety $A$ and sometimes denote it by the same letter $A$. Thus an abelian variety is a couple of its underlying variety and its composition law. An abelian variety is rational over a field $k$ if and only if both the underlying variety of $A$ and the origin 0 of $A$ are rational over $k$.

Let $V$ and $A$ be as above and let $D$ be an $(A \times V)$-divisor. A $V$-divisor $D(a)$ for $a \in A$ is defined by

$$
D \cdot(a \times V)=a \times D(a)
$$

if the intersection on the left side is defined on $A \times V$. When the intersection is not defined, $D(a)$ denotes a $V$-divisor belonging to

$$
C l(D(-x+y+a)+D(x)-D(y))
$$

where $x$ and $y$ are two points on $A$ such that $D(-x+y+a), D(x)$ and $D(y)$ are defined in the old sense. We know that $C l(D(a))$ depends only on the point $a$ and does not depend on the choices of $x$ and $y$ in the latter case. Thus $D(\alpha)$ is defined at any point $a \in A$. If $k$ is a common field of rationality for $V, A$ and $D, D(a)$ is rational over $k(a)$ in the former case and $D(a)$ can be chosen such that it is rational over $k(\alpha)$ in the latter case if $V$ has a $k$-national simple point. Especially if $V$ is an abelian variety too, an $A$-divisor ${ }^{t} D(b)$ for $b \in V$ is defined by

$$
D \cdot(A \times b)={ }^{t} D(b) \times b
$$

if the intersection is defined on $A \times V$; the definition of ${ }^{t} D(b)$ is generalized in the same way as that of $D(a)$.

Let $V$ be now a complete variety non-singular in codimension 1, rational over a field $k$ and having a $k$-rational simple point; then we have a Picard variety $(\hat{V}, D)$ of $V$, rational over $k$ where $\hat{V}$ is an abelian variety rational over $k$ and $D$ is a $(\hat{V} \times V)$-divisor rational over $k$, which is called a Poincaré divisor of $V$ [4]. If we define a map $\theta$ of $\hat{V}$ to $\mathscr{D}_{a}(V) / \mathscr{D}_{\ell}(V)$ by $\theta(x)=C l(D(x)-D(0)), \theta$ is a group isomorphism. A group homomorphism $\Phi_{V}$ (or simply $\Phi$ ) of $\mathscr{D}_{a}(V)$ onto $\hat{V}$ is defined by $\Phi_{V}=\theta^{-1} \circ \pi$ where $\pi$ is a natural surjection of $\mathscr{D}_{a}(V)$ to $\mathscr{D}_{a}(V) / \mathscr{D}_{\ell}(V)$ and 
obviously the kernel of $\Phi_{V}$ is equal to $\mathscr{D}_{\ell}(V)$. We consider a special case where $V$ is an abelian variety and denoted by $A$. It is trivially true that a map of $\hat{A}$ defined by $\hat{x} \rightarrow \Phi(D(\hat{x})-D(0))$ for $\hat{x} \in \hat{A}$ is the identity automorphism $1_{\hat{A}}$ of $\hat{A}$ and furthermore we know that a map $\kappa_{A}$ of $A$ to $\hat{A}$ defined by $\kappa_{A}(x)=\Phi_{\hat{A}}\left({ }^{t} D(x)-{ }^{t} D(0)\right)$ for $x \in A$ is an isomorphism [9]. For an $A$-divisor $X$, a homomorphism $\varphi_{X}$ of $A$ to $\hat{A}$ is defined by $\varphi_{X}(x)=\Phi\left(X_{x}-X\right)$ where $X_{x}$ is the image of $X$ by a translation map of $A: z \mapsto z+x$. For two $A$-divisors $X$ and $Y, \varphi_{X}=\varphi_{Y}$ if and only if $X$ is algebraically equivalent to $Y$. Let $B$ be another abelian variety rational over $k$, let $\lambda$ be a homomorphism of $A$ to $B$, and let $Y$ be a $B$-divisor. An $A$-divisor $\lambda^{-1}(Y)$ is defined in the usual way when the intersection $\Gamma_{\lambda} \cdot(A \times Y)$ is defined on $A \times B$ where $\Gamma_{\lambda}$ is the graph of $\lambda$; and if $\lambda^{-1}(Y)$ is not defined in the usual way, $\lambda^{-1}(Y)$ denotes a divisor belonging to $C l\left(\lambda^{-1}\left(Y^{\prime}\right)\right)$ where $Y^{\prime}$ is a $B$-divisor linearly equivalent to $Y$ such that $\lambda^{-1}\left(Y^{\prime}\right)$ is defined in the usual way. If $Y$ and $\lambda$ are rational over $k, \lambda^{-1}(Y)$ is rational over $k$ when it is defined in the usual way; and when $\lambda^{-1}(Y)$ is defined in the generalized way $\lambda^{-1}(Y)$ can be chosen as a divisor rational over $k$. The dual homomorphism $\hat{\lambda}$ of $\lambda$ is a map of $\hat{B}$ to $\hat{A}$ which is defined by $\hat{\lambda}\left(\Phi_{B}(Y)\right)=\Phi_{A}\left(\lambda^{-1}(Y)\right)$ for any $Y \in \mathscr{D}_{a}(B)$. Especially for a homomorphism $\varphi_{X}$ of $A$ to $\hat{A}$ defined above, we have an equality $\hat{\varphi}_{X} \circ \kappa_{A}=\varphi_{X}$. Let $A_{1}$ be another abelian variety rational over $k$ and let $\left(\hat{A}_{1}, D_{1}\right)$ be a Picard variety of $A_{1}$ rational over $k$. A map $\xi$ of $\mathscr{D}_{a}\left(A \times A_{1}\right) / \mathscr{D}_{\ell}\left(A \times A_{1}\right)$ to $\mathscr{D}_{a}(A) / \mathscr{D}_{\ell}(A) \times \mathscr{D}_{a}\left(A_{1}\right) / \mathscr{D}_{\ell}\left(A_{1}\right)$ defined by $\xi(C l(\bar{X}))=C l\left({ }^{t} \bar{X}(0)\right) \times C l(\bar{X}(0))$ for any $\bar{X} \in \mathscr{D}_{a}\left(A \times A_{1}\right)$ is an isomorphism and if we denote, by $\bar{D}$ (resp. $\bar{D}_{1}$ ), a divisor on $\hat{A} \times \hat{A}_{1} \times A \times A_{1}$ which is the image of a divisor $D \times \hat{A}_{1} \times A_{1}$ (resp. $\hat{A} \times A \times D_{1}$ ) by a permutation isomorphism of $\hat{A} \times A \times \hat{A}_{1} \times A_{1}$ to $\hat{A} \times \hat{A}_{1} \times A \times A_{1}$, then $\left(\hat{A} \times \hat{A}_{1}\right.$, $\left.\bar{D}+\bar{D}_{1}\right)$ is a Picard variety of $A \times A_{1}$ rational over $k$. The homomorphism $\Phi_{A \times A_{1}}$ of $\mathscr{D}_{a}\left(A \times A_{1}\right)$ to $\hat{A} \times \hat{A}_{1}$ is given by $\Phi_{A \times A_{1}}(\bar{X})=\Phi_{A}\left({ }^{t} \bar{X}(0)\right) \times$ $\Phi_{A_{1}}(\bar{X}(0))$ for any $\bar{X} \in \mathscr{D}_{a}\left(A \times A_{1}\right)$.

\section{§ 1. $F M$-structures.}

We start with defining $F M$-systems $\subseteq$ over a field $F$ and $F M$-structures $S$ in $\widetilde{S}$, where we can discuss the field of moduli for $S$.

DEFINITION 1.0. Let $F$ be a field; let $\subseteq$ be a collection of geometric objects $S, S^{\prime}, \cdots$, together with the following three laws: 
i) Given an object $S$ and a field $k$ containing $F$, it is defined whether or not $k$ is a field of rationality for $S$. (When $k$ is a field of rationality for $S$, we say that $S$ is rational over $k$.)

ii) Given two objects $S$ and $S^{\prime}$, it is defined whether or not $S$ is isomorphic to $S^{\prime}$ (noted by $S \cong S^{\prime}$ ).

iii) Given an object $S$ and an automorphism $\sigma$ in $\operatorname{Aut}(K / F)$, the

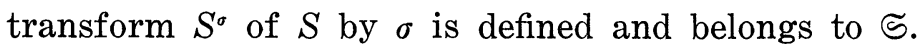

When the pair $(\Im, F)$ satisfies the following conditions, $\subseteq$ is called an $F M$-system over $F$ and an object $S$ in $\subseteq$ is called an $F M$-structure (in $\varsigma$ ).

$\mathrm{fm}$ i) If an object $S$ is rational over a field $k, S$ is rational over any field $k^{\prime}$ containing $k$.

$\mathrm{fm}$ ii) The relation of isomorphy $\cong$ is an equivalence relation.

$f m$ iii) Given $S$ and $S^{\prime}$ in $\subseteq$ and given two automorphisms $\sigma$ and $\tau$ in $\operatorname{Aut}(\boldsymbol{K} / \boldsymbol{F})$, we have

1) $S^{\sigma}=S$ if $\sigma$ acts as the identity on a field of rationality for $S$.

2) $S^{\sigma}$ is rational over $k^{\sigma}$ if $S$ is rational over a field $k$.

3) $S \cong S^{\prime}$ implies $S^{\sigma} \cong S^{\prime}$.

4) $S^{\sigma \tau}=\left(S^{\sigma}\right)^{\tau}$.

When $F$ is the prime field ${ }_{p} F$, $\subseteq$ is called an $F M$-system. Sometimes in this paper we simply say that a geometric object $S$ is an $F M$-structure, without explicit explanation about the $F M$-system $\subseteq$ to which $S$ belongs, when the situation is clear enough.

In many cases, as you see later, an $F M$-structure $S$ is defined by a set of a variety and cycles and a field of rationality for $S$ is a common field of rationality of the variety and some cycles constituting $S$. But still we have another type of $F M$-structures such as PEL-types or especially $C M$-type in the theory of abelian varieties, which are defined and discussed by Shimura and Taniyama [11,12].

1.1. Let $\subseteq$ be an $F M$-system over a field $F$ and let $S$ be an $F M$ structure in $\widetilde{S}$. $A$ subset $G(S, \mathbb{S}$ ) (or simply $G(S)$ ) of $\operatorname{Aut}(\boldsymbol{K} / F)$ is defined by

$$
G(S, \widetilde{S})=\left\{\sigma \in \operatorname{Aut}\left(K / F^{\prime}\right) \mid S^{\sigma} \cong S\right\}
$$

We can easily see that $G(S, \mathcal{S})$ is a subgroup of Aut $(K / F)$ depending only on $\mathbb{S}$ and the isomorphy class $\langle S\rangle$ of $S$ (from $\mathrm{fm}$ ii) and $\mathrm{fm}$ iii 3,4$)$ ). 
DeFINITION 1.1. Let $k_{S}$ be a field containing $F$. If $k_{S}$ satisfied the following two conditions, it is called the field of moduli for $S$ over $F$ (or simply the field of moduli for $S$ ).

$F M$ 1) For an automorphism $\sigma \in \operatorname{Aut~}(K / F), \sigma$ is contained in $G(S, \mathbb{S})$ if and only if the restriction of $\sigma$ on $k_{S}$ is the identify.

$F M 2)$

$$
k_{S}=\cap K
$$

where $K$ runs over the set of all fields of rationality for all $S^{\prime}$ isomorphic to $S$, (containing $F$ ).

1.1.0. An $F M$-structure $S$ has at most one field of moduli for it. The field of moduli for $S$ depends only on the isomorphy class of $S$ (noted by $\langle S\rangle$ ). In other words, if $S$ and $S^{\prime}$ are isomorphic to each other, a field $k_{0}$ is the field of moduli for $S$ over $F$ if and only if it is the field of moduli for $S^{\prime}$ over $F$. Thus the field of moduli $k_{S}$ for $S$ over $F$ is sometimes called the field of moduli for the isomorphy class $\langle S\rangle$ over $F$ and is denoted by $k_{\langle S\rangle}$.

1.1.1. Let $k_{0}$ be a field containing $F$ and satisfying the condition $F M 1$ for $S$, let $K$ be a field of rationality for $S^{\prime}$ isomorphic to $S$; then $k_{0}$ and $K \cdot k_{0}$ are purely inseparable respectively over $K \cap k_{0}$ and $K$, and especially there exists a purely inseparable extension of $K$ which contains $k_{0}$. In fact, we have only to prove that $k_{0}$ is purely inseparable over $K \cap k_{0}$ because the other parts are straight forward from it. We have

$$
G(K / K)=\left\{\sigma \in \operatorname{Aut}(K)|\sigma| K=1_{K}\right\} \subset G(S)=G\left(K / k_{0}\right) .
$$

This inclusion relation implies

$$
\bigcup_{\nu=0}^{\infty} K^{p^{-\nu}} \supset \bigcup_{\nu=0}^{\infty} k_{0}^{p-\nu}
$$

where $p$ is the characteristic of $K$. Thus for any element $a \in k_{0}$, there exists a non-negative integer $\mu$ such that $a^{p^{\mu}}$ is contained in $K$.

1.1.2. Assume that the characteristic of $K$ is zero. For an $F M$ structure $S$, there is at most one field $k_{0}$ which satisfies the condition $F M 1$, and the field $k_{0}$ (if it exists) is contained in any field of rationality $K$ for any $S^{\prime}$ isomorphic to $S$. Furthermore we can easily see that the field $\cap K$ on the right side of the equality in FM2 is a Galois (especially 
algebraic) extension of $k_{0}$. Thus if there is a field of rationality for $S^{\prime}$ which is a regular extension of $k_{0}$, the field $k_{0}$ satisfies the condition $F M 2$ and becomes the field of moduli for $S$.

1.1.3. Let $S$ be an $F M$-structure. If there exists an $F M$-structure $S^{\prime}$ isomorphic to $S$, which is rational over a finite algebraic extension of the prime field, then we have one and only one field $k_{0}$ which satisfies $F M 1$ for $S$, and the field $\cap K$ on the right side of the equality in FM2 is a Galois extension of $k_{0}$.

1.2. Examples. We start with a rather trivial example.

1.2.1. Let $\subseteq$ be the collection of affine (or projective) varieties. For a variety $V$ in $\widetilde{S}$, a field of rationality for $V$ and the transform $V^{\sigma}$ by an automorphism $\sigma \in \operatorname{Aut}(\boldsymbol{K})$ are defined in the usual way. For two varieties $V$ and $V^{\prime}$ in $\subseteq$, we say that $V$ is isomorphic to $V^{\prime}$ if and only if $V$ is identical with $V^{\prime}$. Then obviously $\subseteq$ becomes an $F M$-system over

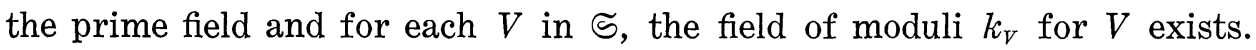
In this case $V$ is rational over $k_{V}$ (see [13]).

1.2.2. Let $V$ be a variety rational over a field $F$; let $\subseteq$ be the total set of cycles on $V$. In the same manner as in 1.2.1 we can define an $F M$-system ( $\subseteq, F)$, especially in this case the isomorphy relation means the identity relation. It can be proved that the field of moduli $k_{X}$ for $X$ over $F$ exists for any $X \in \subseteq$ and $X$ is rational over $k_{X}$ if $X$ is a divisor, but for a cycle of lower dimension the same statement is not true. If the ambient variety $V$ is projective, $k_{X}$ is generated by the Chow point associated to $X$ over $F$. (see [15]).

1.2.3. Let $V$ be a complete variety rational over a field $F$, nonsingular in codimension 1. The field of rationality for a divisor $X \in \mathscr{D}(V)$ and the transform $X^{\sigma}$ of $X$ by an automorphism $\sigma \in \mathrm{A}(\boldsymbol{K} / F)$ are defined in the usual way, and we say that two divisors $X$ and $X^{\prime}$ on $V$ are isomorphic if they are linearly equivalent. Thus $(\mathscr{D}(V), F)$ makes an $F M$ system. Assume that $V$ has an $F$-rational simple point. It is known that there exists the field of moduli $k_{C l(X)}$ for any $X \in \mathscr{D}(V)$ and that there exists a divisor $X^{\prime}$ rational over $k_{C l(X)}$, linearly equivalent to $X$. Let $(\hat{V}, D)$ be a Picard variety of $V$, rational over $F$, and let $\Phi$ be the 
homomorphism of $\mathscr{D}_{a}(V)$ to $\hat{V}$ defined in $\S 0$; then for any divisor $X \in \mathscr{D}_{a}(V)$ we have $k_{C l(X)}=F(\Phi(X)$ ). (see [2,3])

1.2.4. Let $V$ be a projective non-singular variety rational over a field $F$ having an $F$-rational point $P$; let $\mathscr{Z}(V)$ and $\mathscr{Z}_{a}(V)$ be the group of zero-cycles on $V$ and the group of zero-cycles on $V$ which are of degree zero; and let $(\varphi, A(V))$ be an Albanese variety of $V$ rational over $F$ where $A(V)$ is an abelian variety rational over $F$ and $\varphi$ is a map of $V$ to $A(V)$, rational over $F$, with $\varphi(P)=0$. We say that for two cycles $X$ and $X^{\prime}$ in $\mathscr{Z}(V), X$ is isomorphic to $X^{\prime}$ if $X-X^{\prime} \in \mathscr{Z}_{a}(V)$ and $\sum n_{i} \varphi\left(P_{i}\right)=$ $\sum n_{j}^{\prime} \varphi\left(P_{j}^{\prime}\right)$ where $X=\sum n_{i}\left(P_{i}\right)$ and $X^{\prime}=\sum n_{j}^{\prime}\left(P_{j}\right)$. The field of rationality for $X$ and the transform $X^{\sigma}$ of $X$ by $\sigma \in$ Aut $(K / F)$ being defined in the usual way, we have an $F M$-system $(\mathscr{Z}(V), F)$. In this case we can also prove the existence of the field of moduli $k_{C l(X)}$ for $X \in \mathscr{Z}(V)$ and that $k_{C l(X)}$ is the extension of $F$ generated by the point $\sum n_{i} \varphi\left(P_{i}\right)$ on $A(V)$, where $X$ is as above. The problem whether there exists a zero-cycle $X^{\prime}$ isomorphic to $X$ and rational over $k_{C l(X)}$, is not settled so far. (see [1]).

1.2.5. We shall give one more example which is of rather different type from the previous ones. Every detail is proved by Shimura [11] and we follow his definitions and notations without precise description. We consider a $P E L$-type $\Omega$ in the sense of Shimura. The transform $\Omega^{\sigma}$ of $\Omega$ by $\sigma \in A$ ut $(K)$ and the isomorphy between two $P E L$-types are defined in his theory. We say that $\Omega$ is rational over a field $K$ if there is a $P E L$-structure of type $\Omega$ which is rational over $K$. It is proved that $\Omega$ is an $F M$-structure and that the field $k_{\Omega}$ in $[11,5.1]$ is the field of moduli for $\Omega$ in our sense.

On the other hand although a $C M$-type $\Omega^{\prime}$ in the sense [12] is an $F M$-structure in the same manner, the field of moduli for $\Omega^{\prime}$ does not exist in general. Actually the dual field of $\Omega^{\prime}$ satisfies $F M 1$ for $\Omega^{\prime}$ and does not satisfy FM2. In order to treat this case in our line some modifications are needed in the condition $F M 2$, but we do not discuss them.

\section{§ 2. Fields of moduli for $\mathscr{A}$-structures.}

2.0. Let $V$ be a complete non-singular variety; let $\mathscr{D}(V), \mathscr{D}_{a}(V)$ and $\mathscr{D}_{\ell}(V)$ be as in $\S 0$. For a divisor $X \in \mathscr{D}(V), \mathscr{A}(X)$ and $\mathscr{L}(X)$ are the set of positive $V$-divisors algebraically equivalent to $X$ and linearly equivalent 
to $X$ respectively, and $L(X)$ is a set of rational functions on $V$ defined by $L(X)=\{f \mid \operatorname{div}(f)+X \geq 0\}$ where $\operatorname{div}(f)$ implies the $V$-divisor of the function $f$. We know that $L(X)$ is a finite dimensional vector space over the universal domain $K$ and that if $k$ is a common field of rationality for $V$ and $X, L(X)$ admits a linear base $\left(f_{0}, f_{1}, \cdots, f_{l}\right)$, each function $f_{i}$ of which is rational over $k$. If we fix a base $\left(f_{0}, f_{1}, \cdots, f_{l}\right)$ of $L(X)$ as above, we have a rational map $\varphi_{\left(X ; f_{0}, \cdots, f_{l}\right)}$ of $V$ into a projective space $\boldsymbol{P}_{l}$ of dimension $l$ defined by

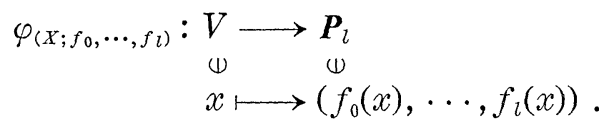

$X$ is called to be very ample if $\mathscr{L}(X)$ has no fixed component and $\varphi_{\left(X ; f_{0}, \cdots, f_{l}\right)}$ is an isomorphism of $V$ onto the image variety, and a divisor $X$ is called to be ample if there is a positive integer $m$ such that $m X$ is very ample. These notations will be used throughout the rest of the paper.

2.1. Under the assumptions and notations in 2.0 , a pair $\mathfrak{A}=(V, \mathscr{A}(X))$ is called an $\mathscr{A}$-structure if $\mathscr{A}(X)$ is an irreducible family of divisors (i.e., an algebraic family of divisors, parametrized by a variety) and any divisor in $\mathscr{A}(X)$ is very ample, and $V$ is called the underlying variety of the $\mathscr{A}$-structure $\mathfrak{U}$. We say that a field $k$ is a field of rationality for $\mathfrak{A}$ if $V$ is rational over $k$ and some $X^{\prime} \in \mathscr{A}(X)$ is rational over $k$. For an automorphism $\sigma \in \operatorname{Aut}(\boldsymbol{K})$, the transform $\mathfrak{X}^{\sigma}$ is defined by $\mathfrak{H}^{\sigma}=\left(V^{\sigma}, \mathscr{A}\left(X^{\sigma}\right)\right)$, which is easily seen to be an $\mathscr{A}$-structure. Let $\mathfrak{U}^{\prime}=\left(V^{\prime}, \mathscr{A}\left(X^{\prime}\right)\right)$ be another $\mathscr{A}$-structure and let $\varphi$ be an isomorphism of $V$ to $V^{\prime}$; then $\varphi$ is called an isomorphism of $\mathfrak{U}$ to $\mathfrak{U}^{\prime}$ if $\varphi$ maps $\mathscr{A}(X)$ to $\mathscr{A}\left(X^{\prime}\right)$. Under these definitions $\mathscr{A}$-structures become $F M$-structures and we can discuss the problem of fields of moduli for $\mathscr{A}$-structures.

Now we consider a condition (A) on an $\mathscr{A}$-structure $\mathfrak{U}=(V, \mathscr{A}(X))$ :

(A) For any $\mathscr{A}$-structure $\mathfrak{Y}^{\prime}=\left(V^{\prime}, \mathscr{A}\left(X^{\prime}\right)\right)$ isomorphic to $\mathfrak{A}$, rational over a field $K$, the irreducible family $\mathscr{A}\left(X^{\prime}\right)$ is rational over $K$.

N.B. We say that the irreducible family $\mathscr{A}\left(X^{\prime}\right)$ is rational over $K$ if the family admits a parameter variety $W$ and a correspondence divisor $\Gamma$ on $W \times V^{\prime}$, both rational over $K$. If $V^{\prime}$ is a projective variety, this condition is equivalent to the fact that the Chow variety $c\left(\mathscr{A}\left(X^{\prime}\right)\right)$ associated with $\mathscr{A}\left(X^{\prime}\right)$ is rational over $K$. The $\mathscr{A}$-structure $\mathscr{U}$ satisfies the 
condition (A) if for any $\mathscr{A}$-structure $\mathfrak{X}^{\prime}=\left(V^{\prime}, \mathscr{A}\left(X^{\prime}\right)\right)$ isomorphic to $\mathfrak{X}$, rational over $K$ and with a projective variety $V^{\prime}$ as its underlying variety, the family $\mathscr{A}\left(X^{\prime}\right)$ is rational over $K$.

Concerning the condition (A) have the following proposition.

Proposition 2.1.1. An $\mathscr{A}$-structure $\mathfrak{U}=(V, \mathscr{A}(X))$ satisfies the condition (A) in either case of the following four cases:

i) $V$ is a curve,

ii) $V$ is the underlying variety of an abelian variety,

iii) the set $\left\{X^{\prime}-X \mid X^{\prime} \in \mathscr{A}(X)\right\}$ covers a complete set of representatives of $\mathscr{D}_{a}(V) / \mathscr{D}_{\ell}(V)$,

iv) the characteristic of the universal domain is zero.

We may assume that $\mathfrak{X}$ is rational over a field of $k$ and $V$ is projective. The case i) is just trivial and case iii) is a theorem of Matsusaka [4, II, Prop. 1]. Under the assumption of $\mathscr{A}$-structures it is easily seen that the Chow variety $c(\mathscr{A}(X))$ is rational over a purely inseparable extension of a field of rationality $k$ for $\mathfrak{A}$. This proves the case iv). In the case ii) if an abelian variety $A$ with the underlying variety $V$ is rational over $k$, it is easy to see that the variety $c(\mathscr{A}(X))$ is rational over $k$. In general case $c(A(X))$ is rational over the field $k(x)$ where $x$ is a generic of point of $V$ over $k$ and since $x$ is an arbitrary generic point of $V$ over $k$, we can conclude that $c(\mathscr{A}(X))$ is rational over $k$.

2.2. Following Matsusaka and Shimura's idea we can prove

THEOREM 2.2. Let $\mathfrak{A}=(V, \mathscr{A}(X))$ be an $\mathscr{A}$-structure satisfying the condition (A). Then the field of moduli $k_{\mathfrak{A}}$ for $\mathfrak{A}$ exists and there is an $\mathscr{A}$-structure $\mathfrak{U}^{\prime}$ isomorphic to $\mathfrak{A}$ and rational over a regular (or a finite separable algebraic) extension of $k_{\mathfrak{x}}$.

Since the theorem is not new but is perfectly proved in $[7,10]$, we shall sketch the idea of the proof which appeared in the literature. Let $k$ be a field of rationality for $\mathfrak{X}$ and let $\bar{X}$ be a generic divisor of $\mathscr{A}(X)$ over $k$, which is rational over a regular extension $k(\bar{x})$ of $k$. Let $\left(f_{0}, f_{1}\right.$, $\left.\cdots, f_{l}\right)$ be a linear base of $L(\bar{X})$ such that each $f_{i}$ is rational over $k(\bar{x})$. Put $\bar{f}_{i}=\sum_{j=0}^{l} u_{i j} f_{j}(i=0,1, \cdots, l)$, where $\left(u_{i j}\right)$ are a set of $(l+1)^{2}$ independent variables over $k(\bar{x})$. $\bar{V}$ denotes the image variety of the map

$$
\varphi_{\left(\bar{X} ; f_{0}, \cdots, \bar{f}_{l}\right)}: V \rightarrow \boldsymbol{P}_{l}
$$


and is a projective variety rational over $k\left(\bar{x}, u_{i j}\right)$, isomorphic to $V$. Let $W$ be the locus of the Chow point $c(\bar{V})$ associated to $\bar{V}$ over $k$. The smallest field of rationality (or the field of moduli in the sense of Example 1.2.1) for $W$ is the field of moduli $k_{\mathfrak{A}}$ for $\mathfrak{A}$.

2.2.1. When $\mathfrak{U}=(A, \mathscr{A}(X))$ is an $\mathscr{A}$-structure where $A$ is the underlying variety of an abelian variety $A$, we can define a new $F M$-structure $\mathfrak{Y}_{0}$ on the same pair $(A, \mathscr{A}(X))$ i.e., a field $k$ is called a field of rationality for $\mathfrak{U}_{0}$ if it is a common field of rationality for the $\mathscr{A}$-structure $\mathfrak{A}$ and the abelian variety $A$; the transform $\mathfrak{A}_{0}^{\sigma}$ of $\mathfrak{U}_{0}$ by $\sigma \in \operatorname{Aut}(K)$ is a pair $\left(A^{\sigma}, \mathscr{A}\left(X^{\sigma}\right)\right)$ where $A^{\sigma}$ is an abelian variety; for another $\mathscr{A}$-structure $\mathfrak{U}_{0}^{\prime}=\left(A^{\prime}, \mathscr{A}\left(X^{\prime}\right)\right)$ with an abelian variety $A^{\prime}$, an isomorphism $\varphi$ of $\mathfrak{U}$ to the $\mathscr{A}$-structure $\mathfrak{U}^{\prime}=\left(A^{\prime}, \mathscr{A}\left(X^{\prime}\right)\right)$ derived from $\mathfrak{H}_{0}^{\prime}$ is called an isomorphism of $\mathfrak{U}_{0}$ to $\mathfrak{U}_{0}^{\prime}$ if $\varphi$ is an isomorphism of the abelian variety $A$ to the abelian variety $A^{\prime}$. Under these definitions the pair $(A, \mathscr{A}(X))$ becomes an $F M$ structure $\mathfrak{I}_{0}$ different from the $\mathscr{A}$-structure $\mathfrak{A}$. We shall call the new $F M$-structure an $\mathscr{A}_{0}$-structure. Thus a pair $(A, \mathscr{A}(X))$ of an abelian variety $A$ and an irreducible family $\mathscr{A}(X)$ of very ample $A$-divisors determines two $F M$-structures- $\mathscr{A}$-structure $\mathfrak{A}$ and $\mathscr{A}_{0}$-structure $\mathfrak{A}_{0}$, and according to Proposition 2.1.1 and Theorem 2.2 we know that the field of moduli $k_{\mathfrak{A}}$ for the $\mathscr{A}$-structure $\mathfrak{A}$ exists.

CoRollary 2.2.2. An $\mathscr{A}_{0}$-structure $\mathfrak{A}_{0}=(A, \mathscr{A}(X))$ admits the field of moduli $k_{\mathfrak{X}_{0}}$ for $\mathfrak{A}_{0}$ and there is an $\mathscr{A}_{0}$-structure isomorphic to $\mathfrak{A}_{0}$ and rational over a regular (or a finite separable algebraic) extension of $k_{\mathfrak{A}_{0}}$. Furthermore if $\mathfrak{U}$ is the $\mathscr{A}$-structure consisting of the same pair $(A, \mathscr{A}(X))$ as $\mathfrak{Y}_{0}$, then $k_{\mathfrak{A}_{0}}$ coincides with the field of moduli $k_{\mathfrak{Q}}$ for $\mathfrak{A}$.

We are going to prove that the field of moduli $k_{\mathfrak{A}}$ for $\mathfrak{A}$ satisfies the conditions $F M 1$ and $F M 2$ for $\mathfrak{A}_{0}$. Let $\mathfrak{U}_{0}^{\prime}=\left(A^{\prime}, \mathscr{A}\left(X^{\prime}\right)\right)$ be another $\mathscr{A}_{0}$-structure. It is easily seen that $(A, \mathscr{A}(X))$ and $\left(A^{\prime}, \mathscr{A}\left(X^{\prime}\right)\right)$ are isomorphic as $\mathscr{A}_{0}$-structure if and only if they are isomorphic as $\mathscr{A}$-structure. This proves $k_{\mathfrak{U}}$ satisfies $F M 1$ for $\mathfrak{U}_{0}$. Concerning the equality $\cap K=k_{\mathfrak{A}}$ where $K$ runs over the set of all fields of rationality of all $\mathfrak{Y}_{0}^{\prime}$ isomorphic to $\mathfrak{A}_{0}$, the inclusion relation $\cap K \supset k_{\mathfrak{A}}$ is obvious from the condition $F M 2$ for $\mathfrak{A}$. To prove the opposite inclusion relation $\cap K \subset k_{\mathfrak{A}}$, it is sufficient to see that if $K^{\prime}$ is a field of rationality for the underlying variety $V$ of an abelian variety, $K^{\prime}=K^{\prime}(x) \cap K^{\prime}(y)$ where $x$ and $y$ are two indepen- 
dent generic points of $V$ over $K^{\prime}$ and that each of $K^{\prime}(x)$ and $K^{\prime}(y)$ is a field of rationality of an abelian variety with underlying variety $V$. This proves that there exists a field of moduli for $\mathfrak{U}_{0}$ and it coincides with $k_{\mathfrak{r}}$. The other part of our corollary is straightforward from Theorem 2.2 and what we have proved above.

2.3. Let $V$ be a complete non-singular variety. It is known that the torsion subgroup $T$ of the group $\mathscr{D}(V) / \mathscr{D}_{a}(V)$ is of finite order [6] and we denote by $t$ the order of $T$. Let $\mathfrak{A}=(V, \mathscr{A}(X))$ be an $\mathscr{A}$-structure with underlying variety $V$. If $(V, \mathscr{A}(m X))$ for a positive integer $m$ is also an $\mathscr{A}$-structure, we denote it by $\mathfrak{X}^{m}$.

Proposition 2.3. Let $\mathfrak{U}=(V, \mathscr{A}(X))$ and $\mathfrak{U}^{m}=(V, \mathscr{A}(m X))$ be two $\mathscr{A}$-structures satisfying the condition (A). Then we have

i) the field of moduli $k_{\mathfrak{A}}$ for $\mathfrak{A}$ is a finite algebraic extension of the field of moduli $k_{\mathfrak{X}^{m}}$ for $\mathfrak{U}^{m}$,

ii) if $m$ is prime to $t, k_{\mathfrak{A}}$ is purely inseparable over $k_{\mathfrak{A}^{m}}$,

iii) if $m$ is prime to tp where $p$ is the characteristic of the universal domain, $k_{\mathfrak{A}}$ coincides with $k_{\mathfrak{U}^{m}}$.

The first assertion i) is proved in [7, Lem. 8]. To prove the second one ii) we have only to see the equality $G(\mathfrak{U})=G\left(\mathfrak{U}^{m}\right)$, which is obvious from the assumption that $m$ is prime to $t$ (as for the definition of $G(\mathfrak{U})$, see 1.1). The third one iii) is a combination of ii) and Lem. 10 in [7].

\section{$\S 3$. Fields of moduli for $\mathscr{P}$-structures (polarized varieties).}

3.0. Let $V$ be a complete non-singular variety and $X$ a divisor on $V$. We denote by $\mathscr{P}(X)$ the set of all positive $V$-divisors $X^{\prime}$ for which there exist two positive integers $m$ and $m^{\prime}$ such that $m X$ is algebraically equivalent to $m^{\prime} X^{\prime}$. A pair $\Re=(V, \mathscr{P}(X))$ of $V$ and $\mathscr{P}(X)$ as above, is called a $\mathscr{P}$-structure (or a polarized variety) if $\mathscr{P}(X)$ contains an ample divisor; and $V$ is called the underlying variety of the $\mathscr{P}$-structure $\mathfrak{B}$ in the case. In the usual way and in the same way as in $\mathscr{A}$-structures (see 2.1) we can define the notion of field of rationality for $\mathfrak{B}$, the transform $\mathfrak{B}^{\circ}$ of $\mathfrak{B}$ by

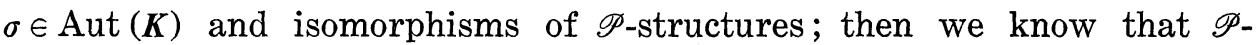
structures become $F M$-structures.

Now we consider a condition (T) on a complete non-singular variety $V$ :

(T) The torsion subgroup of the group $\mathscr{D}(V) / \mathscr{D}_{a}(V)$ is trivial. 
If the underlying variety $V$ of a $\mathscr{P}$-structure $\mathfrak{P}=(V, \mathscr{P}(X))$ satisfies the condition (T), there exists a $V$-divisor $X_{0}$ (not necessarily positive) such that a positive $V$-divisor $X^{\prime}$ belongs to $\mathscr{P}(X)$ if and only if $X^{\prime}$ is algebraic equivalent to $m X_{0}$ with some positive integer $m$. The divisor $X_{0}$ with this property is uniquely determined by the $\mathscr{P}$-structure $\Re$ up to algebraic equivalence and is called a basic polar divisor of $\mathfrak{R}$. It is known that there exists a positive integer $m_{0}$ for $\mathfrak{B}$ such that $\left(V, \mathscr{A}\left(m X_{0}\right)\right)$ is an $\mathscr{A}$ structure satisfying the condition iii) in Proposition 2.1.1 (and hence satisfying the condition (A)) if $m \geq m_{0}[5,7]$.

We consider another condition (P) on a complete non-singular variety $V$ :

(P) Let $V^{\prime}$ be a variety isomorphic to $V$ and rational over a field $K$; then any coset of $\mathscr{D}\left(V^{\prime}\right)$ modulo $\mathscr{D}_{a}\left(V^{\prime}\right)$ has a representative divisor which is rational over a separable algebraic extension of $K$.

Proposition 3.1. A complete non-singular variety $V$ satisfies both conditions $(\mathrm{T})$ and $(\mathrm{P})$ in either of the following two cases

i) $V$ is a curve,

ii) $V$ is the underlying variety of an abelian variety and the characteristic $p$ of the universal domain is not equal to two.

The first case i) of our proposition is obvious. Since the triviality of the torsion subgroup of $\mathscr{D}(V) / \mathscr{D}_{a}(V)$ is known in the case when $V$ is the underlying variety of an abelian variety without any condition on the characteristic (even in the case of characteristic 2) [3,9], it is sufficient to prove that $V$ satisfies the condition (P) in the second case ii). This part of our proposition easily follows the following lemma, which comes from an idea of Mumford [8, p. 121].

Lemma 3.1.1. Let $A$ and $(\hat{A}, D)$ be an abelian variety and its Picard variety, both rational over a field $k$; let $\lambda$ be a homomorphism of $A$ to $\hat{A}$ rational over $k$. Then there exists an $A$-divisor $X$ rational over $k$ such that $\varphi_{X}=\lambda+\hat{\lambda} \circ \kappa_{A}$.

We first consider the following three assertions, where we follow the notations given in $\S 0$ without any comment.

Assertion (I). Let $B$ and $(\hat{B}, E)$ be an abelian variety and its Picard 
variety; let $\alpha$ be a homomorphism of $A$ to $B$; let $Y$ be a B-divisor. Then we have

$$
\varphi_{\alpha-1(Y)}=\hat{\alpha} \circ \varphi_{Y} \circ \alpha
$$

Assertion (II). Let $A_{i}$ and $\left(\hat{A}_{i}, D_{i}\right)$ be an abelian variety and its Picard variety $(i=1,2)$; let $\alpha_{i}$ be a homomorphism of $A$ to $A_{i}(i=1,2) . A$ homomorphism $\alpha$ of $A$ to $A_{1} \times A_{2}$ is defined by $\alpha(x)=\alpha_{1}(x) \times \alpha_{2}(x)$ for $x \in A$. Then the dual homomorphism $\hat{\alpha}$ of $\alpha$ is given by

$$
\hat{\alpha}\left(\hat{x}_{1} \times \hat{x}_{2}\right)=\hat{\alpha}_{1}\left(\hat{x}_{1}\right)+\hat{\alpha}_{2}\left(\hat{x}_{2}\right) \quad \text { for } \hat{x}_{1} \times \hat{x}_{2} \in \hat{A}_{1} \times \hat{A}_{2} .
$$

Assertion (III). Define a divisor $\bar{D}$ on $\hat{A} \times A$ by $\bar{D}=-D+\hat{A} \times$ $D(0)+{ }^{t} D(0) \times A$. Then a homomorphism $\varphi_{\bar{D}}$ of $\hat{A} \times A$ to $\hat{A} \times \hat{A}$ is given by

$$
\varphi_{\bar{D}}(\hat{x} \times x)=\kappa_{A}(x) \times \hat{x} \quad \text { for } \hat{x} \times x \in \hat{A} \times A .
$$

The formula in (I) is well-known [3] and the assertion (II) can easily be proved by recalling the definition of dual homomorphisms and how $\hat{A}_{1} \times \hat{A}_{2}$ is identified with a Picard variety of $A_{1} \times A_{2}$ (see $\S 0$ ). We shall prove only the assertion (III).

For $\hat{x} \times x \in \hat{A} \times A$, we have

$$
\begin{aligned}
\varphi_{D}(\hat{x} \times x) & =\Phi_{\hat{A} \times A}\left(D_{\hat{x} \times x}-D\right) \\
& =\Phi_{\hat{A} \times A}\left(D_{\hat{x} \times x}-D_{\hat{x} \times 0}+D_{\hat{x} \times 0}-D\right) \\
& =\Phi_{\hat{A} \times A}\left(D_{0 \times x}-D\right)+\Phi_{\hat{A} \times A}\left(D_{\hat{x} \times 0}-D\right)
\end{aligned}
$$

We shall determine the first term $\Phi_{\hat{A} \times A}\left(D_{0 \times x}-D\right)$,

$$
\Phi_{\hat{A} \times A}\left(D_{0 \times x}-D\right)=\Phi_{\hat{A}}\left({ }^{t}\left(D_{0 \times x}\right)(0)-{ }^{t} D(0)\right) \times \Phi_{A}\left(D_{0 \times x}(0)-D(0)\right) \quad(\text { see } \S 0)
$$

where

$$
\begin{aligned}
{ }^{t}\left(D_{0 \times x}\right)(0) \times 0 & =D_{0 \times x} \cdot(\hat{A} \times 0)=\{D \cdot(\hat{A} \times(-x))\}_{0 \times x} \\
& =\left\{{ }^{t} D(-x) \times(-x)\right\}_{0 \times x}={ }^{t} D(-x) \times 0
\end{aligned}
$$

and

$$
\begin{aligned}
0 \times D_{0 \times x}(0) & =D_{0 \times x} \cdot(0 \times A)=\{D \cdot(0 \times A)\}_{0 \times x} \\
& =\{0 \times D(0)\}_{0 \times x}=0 \times D(0)_{x} .
\end{aligned}
$$

Thus, we have

$$
\begin{aligned}
\Phi_{\hat{A} \times A}\left(D_{0 \times x}-D\right) & =\Phi_{\hat{A}}\left({ }^{t}\left(D(-x)-{ }^{t} D(0)\right) \times \Phi_{A}\left(D(0)_{x}-D(0)\right)\right. \\
& =-\kappa(x) \times \varphi_{D(0)}(x)
\end{aligned}
$$


In the same way, we have

$$
\Phi_{\hat{A} \times A}\left(D_{\hat{x} \times 0}-D\right)=\varphi_{t_{D(0)}}(\hat{x}) \times(-\hat{x})
$$

Combining the above formulas, we get

$$
\varphi_{D}(\hat{x} \times x)=\left(-\kappa_{A}(x)+\varphi_{t_{D(0)}}(\hat{x})\right) \times\left(\varphi_{D(0)}(x)-\hat{x}\right) .
$$

On the other hand, we easily see that

$$
\varphi_{\hat{A} \times D(0)}(\hat{x} \times x)=0 \times \varphi_{D(0)}(x)
$$

and

$$
\varphi_{t_{D(0) \times A}}(\hat{x} \times x)=\varphi_{t_{D(0)}}(\hat{x}) \times 0
$$

Our formula in (III) is just a direct consequence of the above three formulas.

We are now ready for starting proof of Lemma 3.1.1. Let $\alpha$ be a homomorphism of $A$ to $A \times A$ defined by $\alpha(x)=\lambda(x) \times x$ for $x \in A$ and $\bar{D}$ be a divisor on $\hat{A} \times A$ defined in Assertion (III); then $\alpha$ is rational over $k$, and $\bar{D}$ can be chosen as a divisor rational over $k$. Therefore, we can also choose $\alpha^{-1}(\bar{D})$ as an $A$-divisor rational over $k$ (see $\S 0$ ). According to our three Assertions, we have

$$
\begin{aligned}
\varphi_{\alpha-1(\bar{D})}(x) & =\hat{\alpha} \circ \varphi_{\bar{D}} \circ \alpha(x) \\
& =\hat{\alpha} \circ \varphi_{\bar{D}}(\lambda(x) \times x) \\
& =\hat{\alpha}\left(\kappa_{A}(x) \times \lambda(x)\right) \\
& =\hat{\lambda} \circ \kappa_{A}(x)+\lambda(x)
\end{aligned}
$$

This proves that the divisor $\alpha^{-1}(\bar{D})$ on $A$ is a divisor which we want.

Proof of the last part of Prop. 3.1. Suppose that the underlying variety $V$ of an abelian variety is rational over $k$, and it is sufficient to show that $\mathscr{D}(V) / \mathscr{D}_{a}(V)$ has a complete set of representatives consisting of divisors rational over separable algebraic extensions of $k$. We have an abelian variety $A$ with underlying variety $V$, which is rational over a separable algebraic extension $k^{\prime}$ of $k$. Let $(\hat{A}, D)$ be a Picard variety of $A$, rational over $k^{\prime}$. First we see that for any divisor $X \in \mathscr{D}(A)$, there exists an $A$-divisor $Y$ rational over a separable algebraic extension $k^{\prime \prime}$ of $k$ and algebraically equivalent to $2 X$. In fact, by a Theorem of Chow, a homomorphism $\varphi_{X}$ of $A$ to $\hat{A}$ is rational over a separable algebraic extension $k^{\prime \prime}$ of $k^{\prime}$. Applying Lem. 3.1.1 to our case we have an $A$-divisor 
$Y$ rational over $k^{\prime \prime}$ such that $\varphi_{Y}=\varphi_{X}+\hat{\varphi}_{X} \circ \kappa_{A}=2 \varphi_{X}=\varphi_{2 X}$ and this equality implies that $Y$ is algebraically equivalent to $2 X$ (see $\S 0$.).

For our purpose i.e., the existence of an $A$-divisor $Z$ rational over a separable algebraic extension of $k$ and algebraically equivalent to $X$, we may assume that $X$ is rational over a finite algebraic extension of $k$. Under this assumption there is a positive integer $r$ such that $p^{r} X$ is rational over a separable algebraic extension $k^{(3)}$ of $k$. Since $p$ is not 2 , there are two integers $l$ and $m$ such that $2 l+p^{r} m=1$; and if we put $X_{0}=l Y+m p^{r} X$, the divisor $X_{0}$ is algebraically equivalent to $X$ and rational over the composite $k_{0}$ of $k^{\prime \prime}$ and $k^{(3)}$, which is separable algebraic over $k$. This complets our proof.

THEOREM 3.2. Let $\mathfrak{P}=(V, \mathscr{P}(X))$ be a $\mathscr{P}$-structure where $V$ satisfies both $(\mathrm{T})$ and $(\mathrm{P})$. Then there is a field of moduli $k_{\mathfrak{B}}$ for $\mathfrak{B}$ and there is a $\mathscr{P}$-structure $\mathfrak{P}^{\prime}$ isomorphic to $\mathfrak{P}$ and rational over a regular (or a finite separable algebraic) extension of $k_{\Re}$.

Let $\mathfrak{A}=(V, \mathscr{A}(X))$ be an $\mathscr{A}$-structure satisfying the condition (A) such that its underlying variety $V$ is the same as that of $\mathfrak{B}$ and $\mathscr{A}(X)$ is a subset of $\mathscr{P}(X)$. For brevity, such an $\mathscr{A}$-structure is called a "good $\mathscr{A}$-structure included in $\mathfrak{B} "$. The existence of a good $\mathscr{A}$-structure included in $\mathfrak{P}$ is guaranteed in 3.0. Our proof of Th. 3.2 is divided into two steps: i) the field of moduli $k_{\mathfrak{A}}$ for $\mathfrak{A}$ depends only on $\mathfrak{P}$ and does not depend on the choice of $\mathfrak{X}$ as long as $\mathfrak{A}$ is a good $\mathscr{A}$-structure included in $\mathfrak{B}$, and ii) the field of moduli $k_{\mathfrak{A}}$ for a good $\mathscr{A}$-structure $\mathfrak{A}$ included in $\mathfrak{P}$ is the field of moduli for $\mathfrak{P}$.

Let $\mathfrak{A}=(V, \mathscr{A}(X))$ and $\mathfrak{X}_{1}=\left(V, \mathscr{A}\left(X_{1}\right)\right)$ be two good $\mathscr{A}$-structures included in $\mathfrak{B}$. In order to prove the equality $k_{\mathfrak{Q}}=k_{\mathfrak{N}_{1}}$ we may assume that $X_{1}=m X$ i.e., $\mathfrak{U}_{1}=\mathfrak{U}^{m}$ for a positive integer because the general case can be easily reduced to this special case. Since $k_{\mathscr{V}}$ is purely inseparable extension of $k_{\mathfrak{R}^{m}}$ (see Prop. $2.3 \mathrm{ii}$ )), we have only a to show that there exists a separable extension $K$ of $k_{\mathscr{A}^{m}}$, over which an $\mathscr{A}$-structure $\mathfrak{U}^{\prime}$ isomorphic to $\mathfrak{A}$ is rational. According to Th. 2.2 there is an $\mathscr{A}$ structure $\mathfrak{U}^{\prime \prime}=\left(V^{\prime \prime}, \mathscr{A}\left(m X^{\prime \prime}\right)\right)$ isomorphic to $\mathfrak{U}^{m}$ which is rational over a separable extension $K^{\prime \prime}$ of $k_{\mathfrak{A}^{m}}$. Since the variety $V^{\prime \prime}$ is isomorphic to $V$ and rational over $K^{\prime \prime}$, the condition $(\mathrm{P})$ guarantees that there is a divisor $X^{\prime}$ which is algebraically equivalent to $X^{\prime \prime}$ and rational over a separable extension $K^{\prime}$ of $K^{\prime \prime}$. The pair $\left(V^{\prime \prime}, \mathscr{A}\left(X^{\prime}\right)\right)=\left(V^{\prime \prime}, \mathscr{A}\left(X^{\prime \prime}\right)\right)$ determines an 
$\mathscr{A}$-structure which is isomorphic to $\mathfrak{A}$ and rational over $K^{\prime}$ where $K^{\prime}$ is a separable extension of $k_{\mathfrak{A}^{m}}$. This proves $\mathrm{i}$ ). We are now going to prove the second part ii). Let $\sigma$ be an automorphism of the universal domain $K, \varphi$ an isomorphism of $V$ onto $V^{\sigma}$; then $\varphi$ is an isomorphism of $\mathfrak{A}$ to $\mathfrak{Y}^{\sigma}$ if and only if it is an isomorphism of $\mathfrak{\beta}$ to $\mathfrak{P}^{\sigma}$. In fact the "only if" part is ovbious. Suppose that $\varphi$ is an isomorphism of $\mathfrak{\beta}$ to $\mathfrak{B}^{\circ}$. Let $X_{0}$ be a basic polar divisor of $\mathfrak{\beta}$ and $m$ a positive integer such that $m X_{0}$ is algebraically equivalent to $X$; then $X_{0}^{o}$ is algebraically equivalent to $\varphi\left(X_{0}\right)$ because both $X_{0}^{\sigma}$ and $\varphi\left(X_{0}\right)$ are basic divisors of $\mathfrak{P}^{\sigma}$ and we have

$$
X^{\sigma} \equiv m X_{0}^{\sigma} \equiv m \varphi\left(X_{0}\right) \equiv \varphi\left(m X_{0}\right) \equiv \varphi(X) \quad\left(\bmod \mathscr{D}_{a}\left(V^{\sigma}\right)\right)
$$

This implies that $\varphi$ is an isomorphism of $\mathfrak{A}$ to $\mathfrak{H}^{\sigma}$ and proves the "if" part. This equivalence between two conditions $\mathfrak{A} \cong \mathfrak{U}^{\sigma}$ and $\mathfrak{P} \cong \mathfrak{P}^{\circ}$ induces the equality:

$$
G(\mathfrak{U})=\left\{\sigma \in \operatorname{Aut}(K) \mid \mathfrak{P}^{\sigma} \cong \mathfrak{A}\right\}=\left\{\sigma \in \operatorname{Aut}(\boldsymbol{K}) \mid \mathfrak{P}^{\sigma} \cong \mathfrak{P}\right\}=G(\mathfrak{P}) .
$$

Thus the field $k_{\mathfrak{A}}$ satisfies the condition $F M 1$ for $\mathfrak{P}$. Next we prove that the field $k_{\mathfrak{A}}$ satisfies the condition $F M 2$ for $\mathfrak{P}$. Since a field of rationality for an $\mathscr{A}$-structure isomorphic to $\mathfrak{A}$ is a field of rationality for a $\mathscr{P}$ structure isomorphic to $\mathfrak{B}$, we have only to prove that a field of rationality $K$ for a $\mathscr{P}$-structure $\mathfrak{P}^{\prime}=\left(V^{\prime}, \mathscr{P}\left(X^{\prime}\right)\right)$ isomorphic to $\mathfrak{P}$ is a field of rationality for a good $\mathscr{A}$-structure $\mathfrak{Y}^{\prime}=\left(V^{\prime}, \mathscr{A}\left(X^{\prime \prime}\right)\right)$ included in $\mathfrak{P}^{\prime} . \quad V^{\prime}$ is rational over $K$ and we may assume that $X$ is a $V^{\prime}$-divisor rational over $K$. There is a positive integer $m$ such that a pair $\left(V^{\prime}, \mathscr{A}\left(m X^{\prime}\right)\right)$ determines a good $\mathscr{A}$-structure $\mathfrak{U}^{\prime}$ included in $\mathfrak{P}^{\prime}$, rational over $K$. This proves what we mentioned above and also proves that $k_{\mathfrak{A}}$ satisfies the condition $F M 2$ for $\mathfrak{B}$. Thus the proof of our theorem is completed.

3.2.1. From now on, by a curve we always understand a complete non-singular curve.

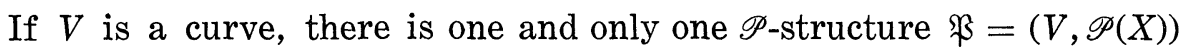
with underlying variety $V$ and $\mathfrak{P}$ admits the field of moduli $k_{\Re}$ (see Prop. 3.1 and Th. 3.2). On the other hand, the notation of fields of rationality for a curve $V$, the transform $V^{\sigma}$ of $V$ by $\sigma \in \operatorname{Aut}(K)$ are defined in the usual way, and "isomorphisms" between two curves mean the usual birational biregular isomorphisms between them. Under these definitions, curves $V$ become $F M$-structures but this new structure $V$ is not different 
from the $\mathscr{P}$-structure $(V, \mathscr{P}(X))$ at all in essential. Thus the field of moduli $k_{(V, \mathscr{F}(X))}$ for $(V, \mathscr{P}(X))$ is at the same time the field of moduli $k_{V}$ for the curve $V$.

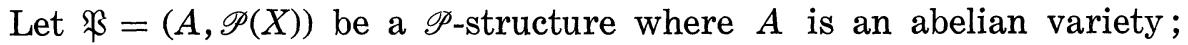
then the field of moduli $k_{\Re}$ exists if the characteristic of the universal domain is not two. On the other hand the pair $(A, \mathscr{P}(X))$ has another kind of $F M$-structure called a $\mathscr{P}_{0}$-structure $\mathfrak{P}_{0}$ (or more customarily a polarized abelian variety), which is defined just in the same way as $\mathscr{A}_{0^{-}}$ structure in 2.2.1.

COROLlary 3.2.2. i) For a curve $V$ the field of moduli $k_{V}$ always exists, and there is a curve $V^{\prime}$ isomorphic to $V$ and rational over a regular (or a finite separable algebraic) extension of $k_{V}$.

ii) Assume that the universal domain is not of characteristic two. For a polarized abelian variety $\mathfrak{B}_{0}=(A, \mathscr{P}(X))$ the field of moduli $k_{\Re_{0}}$ always exists, and there is a polarized abelian variety $\mathfrak{P}_{0}^{\prime}$ isomorphic to $\mathfrak{P}_{0}$ and rational over a regular (or a finite separable algebraic) extension of $k_{\mathfrak{P}_{0}}$. Futhermore if $\mathfrak{P}$ is the $\mathscr{P}$-structure consisting of the same pair $(A, \mathscr{P}(X))$ as $\mathfrak{\beta}_{0}$, then $k_{\Re_{0}}$ coincides with the field of moduli $k_{\Re}$ for $\mathfrak{\Re}$.

The first assertion i) has already been seen above. The second assertion ii) can be proved in the same way as in Cor. 2.2.2.

\section{REFERENCES}

[1] Koizumi, S., On Albanese varieties, Illinois Jour. of Math., 4 (1960) 358-366; II, Memoirs of the College of Sci., Univ. of Kyoto, Series A, 32 (1960) 383-390.

[2 ] Lang, S., Introduction to algebraic geometry, Interscience, New York, 1958.

[ 3 ] Lang, S., Abelian varieties, Interscience, New York, 1959.

[4] Matsusaka, T., On the algebraic construction of the Picard variety, Japanese Jour, of Math., 21 (1951) 217-235; II, ibid., 22 (1952) 51-62.

[5] Matsusaka, T., On algebraic families of positive divisors and their associated varieties on a projective variety, J. Math. Soc. Japan, 5 (1953) 113-136.

[6] Matsusaka, T., The criteria for algebraic equivalence and the torsion group, Amer. J. Math., 79 (1957) 53-66.

[ 7 ] Matsusaka, T., Polarized varieties, fields of moduli and generalized Kummer varieties of polarized abelian varieties, Amer. J. Math., 80 (1958) 45-82.

[ 8 ] Mumford, D., Geometric invariant theory, Ergeb. Math., 34, Springer, 1965.

[9] Nishi, M., the Frobenius theorem and the duality theorem on an abelian variety, Memoirs of the College of Sci., Univ. of Kyoto, Series A, 32 (1960).

[10] Shimura, G., On the theory of automorphic functions, Ann. of Math., 76 (1959) 101-144. 
[11] Shimura, G., Moduli and fibre systems of abelian varieties, Ann. of Math., 83 (1966) 294-338.

[12] Shimura, G. and Taniyama, Y., Complex multiplications of abelian varieties and its applications to number theory, Pub. Math. Soc. Japan, 6, 1961.

[13] Weil, A., Foundations of algebraic geometry, Revised and Enlarged Edition, Publ. AMS. New York, 1962.

[14] Weil, A., Variétés abéliennes et courbes algébriques, Act. Sci. Ind., 1064 Paris: Hermann, 1948.

[15] Weil, A., On algebraic groups of transformations, Amer. J. Math., 77 (1955) $355-391$.

Tokyo University of Education 